\title{
Herpes simplex virus genotyping in neurological abnormalities-clinical relevance for disease monitoring
}

\begin{abstract}
Herpes Simplex Virus (HSV) is an important etiological agent for Neurological disorders like meningitis, seizures etc. Current work includes the molecular reveling of HSV 1 and 2 in CSF specimens. 13 cases of CSF were processed for the typing of HSV by Real Time PCR and out of which 03 cases came positive. All the positive cases were HSV genotype-1. Advanced biotechnological tools promises fast, accurate and timely diagnosis of such viral diseases for further prognosis.
\end{abstract}

Keywords: neurological abnormalities, real time pcr, herpes simplex viruses, genotypes, viral meningitis
Volume 3 Issue 8 - 2016

\author{
Rekha Gorkela, Sachin Kumar, Anchal \\ Chaurasia, Binish Jawed, Mukta Sehgal, \\ Meenakshi Bahuguna,Vatsal Prashar, Indu \\ Bushan, Manish Dev Sharma, Narotam \\ Sharma \\ Department of Biochemistry, India \\ Gurukul Kangari Vishwavidyalaya, India \\ Gurukul Kangri, University, India \\ Department of Biotechnology, Maharaj Vinayak Global \\ University, India
}
Correspondence: Narotam Sharma, Central Molecular Research Laboratory, Department of Biochemistry, Shri Guru Ram Rai Institute of Medical \& Health Sciences, Patel Nagar, Dehradun-24800 I, Uttarakhand, India, Emailsharamanarotam5@gmail.com

Received: October 17, 2016 | Published: December 30, 2016
Abbreviations: HSV, herpes simplex viruses; STI, sexually transmitted infection; CSF, cerebrospinal fluid; PCR, polymerase chain reaction

\section{Introduction}

Herpes simplex viruses (HSV) are categorized into two types, HSV-1, or oral herpes and HSV-2, or genital herpes. HSV-1 is mainly transmitted by oral to oral contact, whereas HSV-2 is a sexually transmitted infection (STI) that can cause painful genital ulcer. ${ }^{1} \mathrm{HSV}-1$ is a highly contagious infection, which is common and endemic throughout the world. The virus causes lifelong infection, and there is no cure, although treatment can reduce symptoms. After the first (primary) infection, HSV, like other herpes viruses, remains dormant (latent) in the body and can periodically reactivate and cause symptoms. ${ }^{2,3}$ HSV infection causes recurring episodes of small, painful, fluid-filled blisters on the skin, mouth, lips (cold sores), eyes, or genitals. ${ }^{4,5}$ In the current study, characterization of HSV genotypes 1 and 2 in cerebrospinal fluid (CSF) in the patients with Viral Meningitis and other Neurological Disorders cases were studied.

\section{Materials and methods}

Cerebrospinal fluid was collected from various Departments of Shri Mahant Indiresh Hospital, Patel Nagar, Dehradun, Uttrakhand. 13 cerebrospinal fluids (CSF) were collected from the patients with Neurological disorders which mainly include seizures, epilepsy, meningitis, hydrocephalus, altered sensonium etc. Further the CSF was preprocessed for DNA isolation and DNA was extracted by silica column method. The amplification of glycoprotein gene in HSV was done by Qiagen Rotor-gene Q Real Time PCR. Pre PCR mix was prepared as per the manufacturer's protocol (artus HSV-1/2 PCR Kit CE-QIAGEN). All the 13 cases were processed for the molecular genotyping of HSV-1 and HSV-2 along with exogenous internal control which is used to check for any artifacts in processing and in PCR protocol. Direct detection of the florescence can be detected as specific amplification plots which can be visualized in channel cycling Green $(470 \mathrm{~nm})$ and cycling Orange (detector 610nm) and Internal Control fluorescence channel cycling Yellow $(530 \mathrm{~nm}-555 \mathrm{~nm})$ for HSV-1, HSV-2 and internal control respectively (Figure 1).

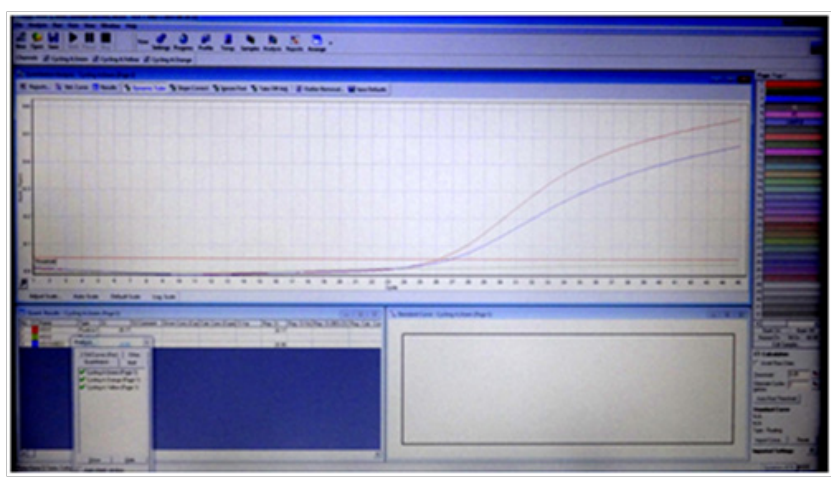

Figure I Amplification plots for HSV-I and 2 in Real time PCR machine.

\section{Results}

Out of 13 CSF specimens processed, 03 cases came positive with the occurrence of HSV type 1 in all the positive cases. As the HSV-1 is prevalent worldwide it was also studied w.r.t to gender and age wise. 10 males and 03 females were considered for the study and HSV-1 positive cases were mostly seen in males. All the positive cases were with meningitis \& Neurological disorders.

\section{Discussion and Conclusion}

HSV-1 is mainly transmitted by oral contact whereas HSV-2 causes sexually transmitted infections. Viral meningitis can cause 
inflammation of the brain (encephalitis). HSV meningitis is a serious infection but diagnosis previously required brain biopsy in certain cases due to low sensitivity of CSF culture and serology. ${ }^{6,7}$ PCR allows the detection of HSV DNA from CSF with 95\% sensitivity thus avoiding invasive brain biopsy. Viral meningitis, commonly caused by either enteroviruses or HSV is more reliably detected by PCR when compared to culture and in a shorter time (one verses up to 5days). HSV 1 classically presents as herpes gingivostomatitis an infection of the oral mucosa. It can also cause conjunctivitis, keratitis, and herpetic whitlow. HSV 2 is most common cause of genital ulcer. It has been documented that as many as one third of herpes infections are due to HSV 1, particularly in adolescent and young adult. ${ }^{8}$ The proper management of such HSV infections required customized therapy with specific regimens. Advanced Molecular tools for specific typing of HSV have now enabled the clinicians to treat the disease effectively.

\section{Acknowledgments}

None.

\section{Conflicts of interest}

Author declares there are no conflicts of interest.

\section{References}

1. Kewal KJ. Molecular Diagnostics for Neurological Disorders. Applications of Biotechnology in Neurology. 2013. p.155-210.

2. Itzhaki RF, Lin WR, Shang D, et al. Herpes simplex virus type 1 in brain and risk of Alzheimer's disease. Lancet. 1997;349(9047):241-244.

3. Kennedy PG. Potential use of herpes simplex virus (HSV) vectors for gene therapy of neurological disorders. Brain. 1997;120(7):1245-1259.

4. Whitley R. Neonatal herpes simplex virus infection. Curr Opin Infect Dis. 2004;17(3):243-246.

5. Tyler KL. Herpes simplex virus infections of the central nervous system: encephalitis and meningitis, including Mollaret's. Herpes. 2004;11(2):57A-64A.

6. Whitley RJ, Lakeman F. Herpes simplex virus infections of the central nervous system: therapeutic and diagnostic considerations. Clin Infect Dis. 1995;20(2):414-420.

7. Kimberlin D. Herpes simplex virus, meningitis and encephalitis in neonates. Herpes. 2004;11(2):65A-76A.

8. Langenberg AG, Corey L, Ashley RL, et al. A prospective study of new infections with herpes simplex virus type 1 and type 2. N Engl J Med. 1999;341(19):1432-1438.

\section{Funding}

None. 